\title{
Robustness of retrieval properties against imbalance between long-term potentiation and depression of spike-timing-dependent plasticity
}

\author{
Narihisa Matsumoto 1,2 , 因 and Masato Okada ${ }^{2,3,0}$ \\ ${ }^{1}$ Graduate School of Science and Engineering, Saitama University, Saitama 338-8570, Japan \\ ${ }^{2}$ RIKEN Brain Science Institute, Saitama 351-0198, Japan \\ 3 "Intelligent Cooperation and Control", PRESTO, JST, Saitama 351-0198, Japan
}

(Dated: October 25, 2018)

\begin{abstract}
Spike-timing-dependent plasticity (STDP) has recently been shown in some physiological studies. STDP depends on the precise temporal relationship of pre- and post-synaptic spikes. Many authors have indicated that a precise balance between long-term potentiation (LTP) and long-term depression (LTD) of STDP is significant for a stable learning. However, a situation in which the balance is maintained precisely is inconceivable in the brain. Using a method of the statistical neurodynamics, we show robust retrieval properties of spatio-temporal patterns in an associative memory model against the imbalance between LTP and LTD. When the fluctuation of LTD is assumed to obey a Gaussian distribution with mean 0 and variance $\delta^{2}$, the storage capacity takes a finite value even at large $\delta$. This means that the balance between LTP and LTD of STDP need not to be maintained precisely, but must be maintained on average. Furthermore, we found that a basin of attraction becomes smaller as $\delta$ increases while an initial critical overlap remains unchanged.
\end{abstract}

PACS numbers: 87.18.Sn, 89.70.+c, 05.90.+m

\section{INTRODUCTION}

Recent experimental finding indicates that synaptic modification in cortical neurons depends on the precise temporal relationship between pre- and post-synaptic spikes 1, 2, 3]. In particular, pre-synaptic spikes that precede post-synaptic firing induce long-term potentiation (LTP) by no more than $20 \mathrm{~ms}$, while those that follow post-synaptic firing induce long-term depression (LTD), with a rapid transition (a few ms). The magnitude of synaptic modification decays exponentially with the time interval between pre- and post-synaptic spikes. This form of synaptic modification has been called spiketiming-dependent plasticity (STDP) 4 or temporally asymmetric Hebbian learning (TAH) [5, 6].

The functional role of STDP has been investigated by many authors. They showed that STDP is a mechanism for synaptic competition [4, 5, 6, 7, 8, 9] or a learning mechanism of sequential patterns $10,11,12,13,14,15$, 16. Asymmetric learning window depending on spike timing like STDP has been studied and shown to be appropriate learning rule for sequential patterns 17, 18, 19]. However, this asymmetric learning rule does not involve LTD. Some authors showed that the balance between LTP and LTD of STDP is significant for a stable learning [4, 14, 15, 16]. In our previous work, we analytically showed that STDP has the same qualitative effect as the covariance rule when the spatio-temporal patterns are stored since the differences between spike times that induce LTP or LTD are capable of canceling out the effect of the firing rate information [16]. In the brain, a situation in which the balance is maintained precisely is inconceivable. The data points obtained by experiments are fluctuated in the different trials [1, 3]. Therefore, it is meaningful to discuss more biological plausible situation to investigate the neuronal mechanism for sequential learning in the brain. Some authors numerically investigated the impact of the imbalance between LTP and LTD on the network properties [4, 14].

The aim of this paper is to analytically discuss the retrieval properties of spatio-temporal patterns in an associative memory model that incorporates the imbalance between LTP and LTD of STDP using a method of the statistical neurodynamics [16, 20, 21]. According to our previous work, when the balance is not precisely maintained, it is impossible to cancel out the information of firing rate. Consequently, a cross-talk noise diverges. However, if the magnitudes of LTP and LTD are equivalent on average in a learning process, it may be possible to stably retrieve spatio-temporal patterns. Since the ratio of LTP and LTD is crucial, the magnitude of LTD changes while that of LTP is fixed. We found that the storage capacity takes a finite value even at large $\delta$ when the fluctuation of LTD is assumed to obey a Gaussian distribution with mean 0 and variance $\delta^{2}$. This implies that the balance between LTP and LTD of STDP need not to be maintained precisely, but must be maintained on average. This mechanism might work in the brain. Furthermore, we found that a basin of attraction becomes smaller as $\delta$ increases while an initial critical overlap remains unchanged.

\section{MODEL}

The model contains $N$ binary neurons with reciprocal connections. Each neuron has a binary state $\{0,1\}$. 
We define discrete time steps and the following rule for synchronous updating:

$$
\begin{aligned}
u_{i}(t) & =\sum_{j=1}^{N} J_{i j} x_{j}(t), \\
x_{i}(t+1) & =F\left(u_{i}(t)-\theta\right), \\
F(u) & = \begin{cases}1 . & u \geq 0 \\
0 . & u<0,\end{cases}
\end{aligned}
$$

where $x_{i}(t)$ is the state of the $i$-th neuron at time $t, u_{i}(t)$ is the internal potential of that neuron, and $\theta$ is a uniform threshold. If the $i$-th neuron fires at $t$, its state is $x_{i}(t)=1$; otherwise, $x_{i}(t)=0 . J_{i j}$ is the synaptic weight from the $j$-th neuron to the $i$-th neuron. Each element $\xi_{i}^{\mu}$ of the $\mu$-th memory pattern $\boldsymbol{\xi}^{\mu}=\left(\xi_{1}^{\mu}, \xi_{2}^{\mu}, \cdots, \xi_{N}^{\mu}\right)$ is generated independently by

$$
\operatorname{Prob}\left[\xi_{i}^{\mu}=1\right]=1-\operatorname{Prob}\left[\xi_{i}^{\mu}=0\right]=f .
$$

The expectation of $\boldsymbol{\xi}^{\mu}$ is $\mathrm{E}\left[\xi_{i}^{\mu}\right]=f$, and thus $f$ is considered to be the mean firing rate of the memory pattern. The memory pattern is sparse when $f \rightarrow 0$, and this coding scheme is called sparse coding.

The synaptic weight $J_{i j}$ follows the form of synaptic plasticity, which depends on the difference in spike times between the $i$-th (post-) and $j$-th (pre-) neurons. The time difference determines whether LTP or LTD is induced. This type of learning rule is called spike-timingdependent plasticity (STDP). The biological experimental findings show that LTP or LTD is induced when the difference in the pre- and post-synaptic spike times falls within about $20 \mathrm{~ms}$ [3]. We define a single time step in equations (1] 3) as $20 \mathrm{~ms}$, and durations within $20 \mathrm{~ms}$ are ignored. The learning rule based on STDP conforms to this equation:

$$
J_{i j}=\frac{1}{N f(1-f)} \sum_{\mu=1}^{p}\left\{\xi_{i}^{\mu+1} \xi_{j}^{\mu}-\left(1+\epsilon_{i j}^{\mu-1}\right) \xi_{i}^{\mu-1} \xi_{j}^{\mu}\right\} .
$$

The number of memory patterns is $p=\alpha N$, where $\alpha$ is defined as a loading rate. LTP is induced when the $j$-th neuron fires one time step before the $i$-th neuron, $\xi_{i}^{\mu+1}=$ $\xi_{j}^{\mu}=1$, while LTD is induced when the $j$-th neuron fires one time step after the $i$-th neuron, $\xi_{i}^{\mu-1}=\xi_{j}^{\mu}=1$. Since the ratio of LTP and LTD is crucial, the magnitude of LTD changes while the magnitude of LTP and the time duration are fixed. $\epsilon_{i j}^{\mu}$ is generated independently and obeys a Gaussian distribution with mean $\epsilon$ and variance $\delta^{2}: \epsilon_{i j}^{\mu} \sim \mathcal{N}\left(\epsilon, \delta^{2}\right)$. Fig [1] shows the time function of STDP in our model. When $\epsilon_{i j}^{\mu}=0$, the balance between LTP and LTD is precisely maintained and then the model is equivalent to the previous model [16]. A sequence of $p$ memory patterns is stored by STDP: $\boldsymbol{\xi}^{1} \rightarrow \boldsymbol{\xi}^{2} \rightarrow \cdots \rightarrow$ $\boldsymbol{\xi}^{p} \rightarrow \boldsymbol{\xi}^{1} \rightarrow \cdots$. In other words, $\boldsymbol{\xi}^{1}$ is retrieved at $t=1$, $\boldsymbol{\xi}^{2}$ is retrieved at $t=2$, and $\boldsymbol{\xi}^{1}$ is retrieved at $t=p+1$. There is a critical value $\alpha_{C}$ of the loading rate, so that the loading rate larger than $\alpha_{C}$ makes retrieval of the pattern sequence unstable. $\alpha_{C}$ is called a storage capacity.

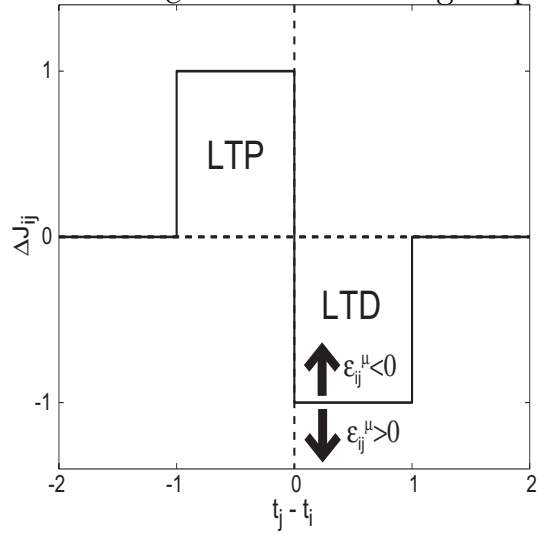

FIG. 1: The time function of STDP in our model. LTP is induced when the $j$-th neuron fires one time step before the $i$-th one. LTD is induced when the $j$-th neuron fires one time step after the $i$-th one. $\epsilon_{i j}^{\mu}$ follows a Gaussian distribution with mean $\epsilon$ and variance $\delta^{2}$.

\section{THEORY}

In this section, we derive dynamical equations that describe the retrieval properties of the network. In this paper, we consider the thermodynamic limit: $N \rightarrow \infty$. The $i$-th neuronal internal potential $u_{i}(t)$ at time $t$ can be expressed as

$$
\begin{aligned}
u_{i}(t) & =\sum_{j=1}^{N} J_{i j} x_{j}(t)=\frac{1}{N f(1-f)} \sum_{j=1}^{N} \sum_{\mu=1}^{p}\left\{\xi_{i}^{\mu+1} \xi_{j}^{\mu}-\left(1+\epsilon_{i j}^{\mu-1}\right) \xi_{i}^{\mu-1} \xi_{j}^{\mu}\right\} x_{j}(t) \\
& =\frac{1}{N f(1-f)} \sum_{j=1}^{N} \sum_{\mu=1}^{p}\left(\xi_{i}^{\mu+1} \xi_{j}^{\mu}-\xi_{i}^{\mu-1} \xi_{j}^{\mu}\right) x_{j}(t)-\frac{1}{N f(1-f)} \sum_{j=1}^{N} \sum_{\mu=1}^{p} \epsilon_{i j}^{\mu-1} \xi_{i}^{\mu-1} \xi_{j}^{\mu} x_{j}(t) .
\end{aligned}
$$

Using the periodic boundary condition of $\xi_{i}^{p+1}=\xi_{i}^{1}$ and $\xi_{i}^{0}=\xi_{i}^{p}, \sum_{\mu=1}^{p}\left(\xi_{i}^{\mu+1} \xi_{j}^{\mu}-\xi_{i}^{\mu-1} \xi_{j}^{\mu}\right)=\sum_{\mu=1}^{p}\left(\bar{\xi}_{i}^{\mu+1} \bar{\xi}_{j}^{\mu}-\right.$ $\left.\bar{\xi}_{i}^{\mu-1} \bar{\xi}_{j}^{\mu}\right)$ with $\bar{\xi}_{i}^{\mu}=\xi_{i}^{\mu}-f$. Using this relationship, $u_{i}(t)$ 
is given by

$$
\begin{aligned}
u_{i}(t)= & \frac{1}{N f(1-f)} \sum_{j=1}^{N} \sum_{\mu=1}^{p}\left(\bar{\xi}_{i}^{\mu+1}-\bar{\xi}_{i}^{\mu-1}\right) \bar{\xi}_{j}^{\mu} x_{j}(t) \\
& -\frac{1}{N f(1-f)} \sum_{j=1}^{N} \sum_{\mu=1}^{p} \epsilon_{i j}^{\mu-1} \xi_{i}^{\mu-1} \xi_{j}^{\mu} x_{j}(t) \\
= & \sum_{\mu=1}^{p}\left(\bar{\xi}_{i}^{\mu+1}-\bar{\xi}_{i}^{\mu-1}\right) m^{\mu}(t) \\
& -\frac{1}{N f(1-f)} \sum_{j=1}^{N} \sum_{\mu=1}^{p} \epsilon_{i j}^{\mu-1} \xi_{i}^{\mu-1} \xi_{j}^{\mu} x_{j}(t) \\
= & \left(\bar{\xi}_{i}^{t+1}-\bar{\xi}_{i}^{t-1}\right) m^{t}(t)+\sum_{\mu \neq t}^{p}\left(\bar{\xi}_{i}^{\mu+1}-\bar{\xi}_{i}^{\mu-1}\right) m^{\mu}(t) \\
& -\frac{1}{N f(1-f)} \sum_{j=1}^{N} \sum_{\mu=1}^{p} \epsilon_{i j}^{\mu-1} \xi_{i}^{\mu-1} \xi_{j}^{\mu} x_{j}(t)
\end{aligned}
$$

where $m^{\mu}(t)$ is an overlap between $\boldsymbol{\xi}^{\mu}(t)$ and $\boldsymbol{x}(t)$ and is given by

$$
m^{\mu}(t)=\frac{1}{N f(1-f)} \sum_{i=1}^{N} \bar{\xi}_{i}^{\mu} x_{i}(t) .
$$

The first term in equation (10) is a signal term for the retrieval of the target pattern $\boldsymbol{\xi}^{t+1}$. The second and third terms are a cross-talk noise term that represents contributions from non-target patterns other than $\boldsymbol{\xi}^{t-1}$ and that prevents $\boldsymbol{\xi}^{t+1}$ from being retrieved. The third term is also a compensation term originated by a deviation from the balance between LTP and LTD of STDP. Since this term is order of $N$ with respect to $N, O(N)$, when $\epsilon \neq 0$, it diverges in the thermodynamic $N$ limit: $N \rightarrow \infty$. This means that the stored limit cycle using the present learning rule (equation (5) is unstable in the limit of $N \rightarrow \infty$ when $\epsilon \neq 0$. Therefore, we will discuss the $\epsilon=0$ case: $\epsilon_{i j}^{\mu} \sim \mathcal{N}\left(0, \delta^{2}\right)$.

We derive the dynamical equations using the method of the statistical neurodynamics [16, 20, 21]. When it is possible to store a pattern sequence, a cross-talk noise term, that is, the second and third terms in equation (10) is assumed to obey a Gaussian distribution with the average 0 and time-dependent variance $\sigma^{2}(t)$ 16, 20]. We derive the recursive equations for $m^{t}(t)$ and $\sigma^{2}(t)$ to investigate whether the memory pattern $\boldsymbol{\xi}^{t}$ is retrieved or not. Since $m^{t}(t)$ depends on $\sigma^{2}(t)$, we derive $\sigma^{2}(t)$. The dynamical equations are derived as

$$
\begin{aligned}
m^{t}(t)= & \frac{1-2 f}{2} \operatorname{erf}\left(\phi_{0}\right)-\frac{1-f}{2} \operatorname{erf}\left(\phi_{1}\right)+\frac{f}{2} \operatorname{erf}\left(\phi_{2}\right), \\
\sigma^{2}(t)= & \sum_{a=0}^{t} 2(a+1) \mathrm{C}_{(a+1)} \alpha q(t-a) \prod_{b=1}^{a} U^{2}(t-b+1) \\
& +\frac{\alpha \delta^{2}}{(1-f)^{2}} q(t),
\end{aligned}
$$

$$
\begin{aligned}
U(t)= & \frac{1}{\sqrt{2 \pi} \sigma(t-1)}\left\{\left(1-2 f+2 f^{2}\right) e^{-\phi_{0}^{2}}\right. \\
& \left.+f(1-f)\left(e^{-\phi_{1}^{2}}+e^{-\phi_{2}^{2}}\right)\right\}, \\
q(t)= & \frac{1}{2}\left\{1-\left(1-2 f+2 f^{2}\right) \operatorname{erf}\left(\phi_{0}\right)-f(1-f)\left(\operatorname{erf}\left(\phi_{1}\right)\right.\right. \\
& \left.\left.+\operatorname{erf}\left(\phi_{2}\right)\right)\right\} .
\end{aligned}
$$

where $\operatorname{erf}(y)=\frac{2}{\sqrt{\pi}} \int_{0}^{y} \exp \left(-u^{2}\right) d u$, and $\phi_{0}=\frac{\theta}{\sqrt{2} \sigma(t-1)}$, $\phi_{1}=\frac{-m^{t-1}(t-1)+\theta}{\sqrt{2} \sigma(t-1)}, \phi_{2}=\frac{m^{t-1}(t-1)+\theta}{\sqrt{2} \sigma(t-1)},{ }_{b} \mathrm{C}_{a}=\frac{b !}{a !(b-a) !}$ and $a$ ! is the factorial with positive integer $a$. The detail derivation of the dynamical equations is shown in Appendix A.

\section{RESULTS}

Fig[2] shows the dependence of the overlap $m^{t}(t)$ on the loading rate $\alpha$ when the mean firing rate of the memory pattern is $f=0.1$, and the threshold is $\theta=0.52$, which is optimized to maximize the storage capacity. (a) is the case at $\delta=0.0,(\mathrm{~b})$ is at $\delta=1.0$, and (c) is at $\delta=2.0$. The lines show analytical results obtained by the dynamical equations (12 (15). The upper line denotes the steady-state values of the overlap $m^{t}(t)$ in retrieval of the pattern sequence. $m^{t}(t)$ is obtained by setting the initial state of the network at the first memory pattern: $\boldsymbol{x}(1)=\boldsymbol{\xi}^{1}$. Setting the initial values at $m^{1}(1)=1$, $\sigma^{2}(1)=2 \alpha f+\frac{\alpha \delta^{2} f}{(1-f)^{2}}, U(1)=0$ and $q(1)=f$ and using the dynamical equations (12) 15), $m^{t}(t)$ is obtained. When the overlap at the steady state is smaller than 0.5 , the critical loading rate $\alpha$ is regarded as the storage capacity $\alpha_{C}$. The storage capacity $\alpha_{C}$ is $0.27(\mathrm{a}), 0.178(\mathrm{~b})$, and $0.087(\mathrm{c})$. The lower line indicates the dependence of an initial critical overlap $m_{C}$ on $\alpha$. The stored pattern sequence is retrievable when the initial overlap $m^{1}(1)$ is greater than the critical value $m_{C}$. The region in which $m^{1}(1)$ is larger than $m_{C}$ represents the basin of attraction for the retrieval of the pattern sequence. $m_{C}$ is obtained by setting the initial state of the network at $\boldsymbol{\xi}^{1}$ with additional noise. We employ the following method to add noise. $100 s \%$ of the minority components $\left(x_{i}(1)=1\right)$ are flipped, while the same number of majority components $\left(x_{i}(1)=0\right)$ are also flipped. The initial overlap $m^{1}(1)$ is given as $1-\frac{2 s}{1-f}$. Then the mean firing rate of the network is kept equal to that of the memory pattern, $f$. The other initial values are equivalent to the upper line case. When the overlap at the steady state is smaller than 0.5 , the initial overlap $m^{1}(1)$ is regarded as the initial critical overlap $m_{C}$. The data points and error bars indicate the results of computer simulations of 11 trials with 5000 neurons: $N=5000$. The results are obtained from the equations (115). The data points indicate median values and both ends of the error bars does $1 / 4$ and $3 / 4$ deviations. A discrepancy between the values of $m_{C}$ obtained by the computer simulations and the analytical results 

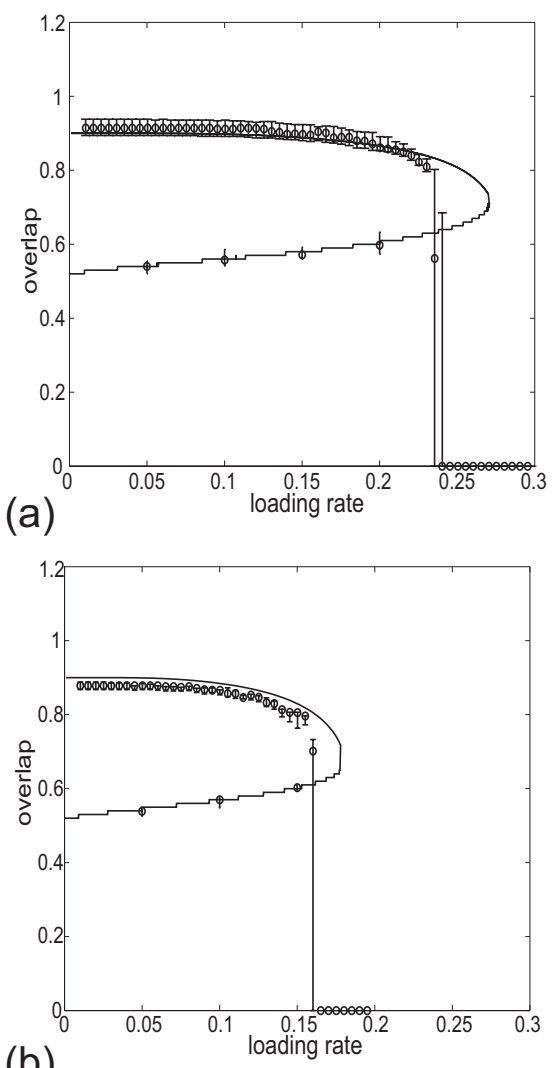

(b)

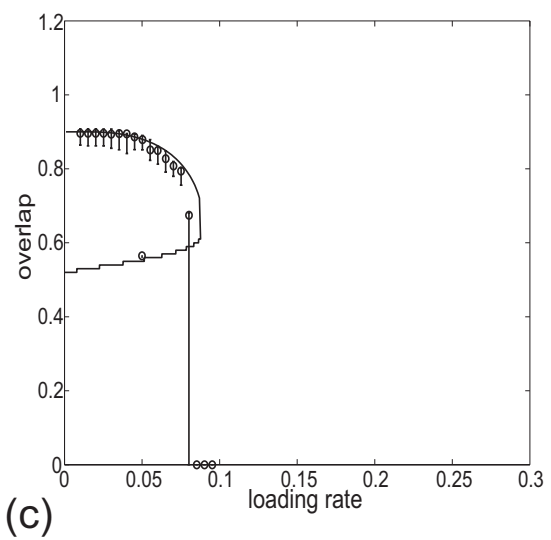

FIG. 2: The dependence of $\alpha_{C}$ and the basin of attraction on the loading rate at $f=0.1$ and $\theta=0.52$. The lower line represents the initial critical overlap and the upper line does the overlap at the steady state. The data points and the error bars show the results of computer simulation of 11 trials at $N=5000$. (a): $\delta=0.0,(\mathrm{~b}): \delta=1.0,(\mathrm{c}): \delta=2.0$. $\alpha_{C}=0.27(\mathrm{a}), 0.178(\mathrm{~b}), 0.087(\mathrm{c})$. The basin of attraction decreases as $\delta$ increases.

is originated from the finite size effect of the computer simulations [16].

Fig 3 shows the dependence of the storage capacity $\alpha_{C}$ on the standard derivation $\delta$ of the fluctuation of LTD at $f=0.1$ and $\theta=0.52$. The solid line shows the analytical results obtained by the same procedure to obtain $\alpha_{C}$ in

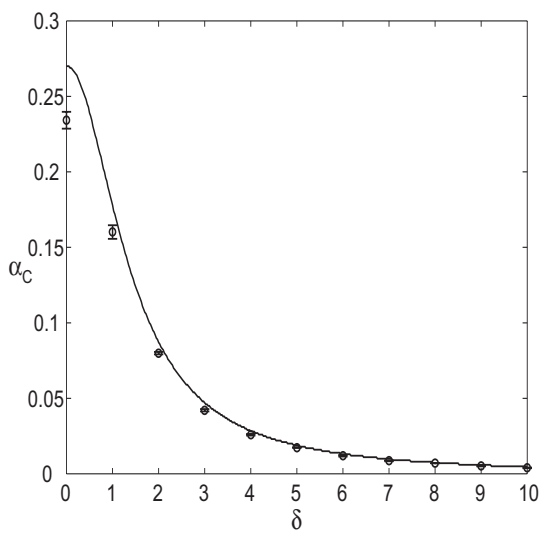

FIG. 3: The dependence of the storage capacity $\alpha_{C}$ on $\delta$. The solid line shows the analytical results. The data points and error bars show the results of computer simulation of 10 trials at $N=5000$. Both results are obtained at $f=0.1$ and $\theta=0.52$. As the variance $\delta^{2}$ of the fluctuation increases, $\alpha_{C}$ decreases.

Fig 2. The data points and error bars show the results of computer simulation of 10 trials at $N=5000$. The means and standard deviations of $\alpha_{C}$ of 10 trials are plotted as the data points and the error bars, respectively. As the variance $\delta^{2}$ increases, $\alpha_{C}$ decreases. In other words, the model is robust against the imbalance between LTP and LTD of STDP. Thus, the balance does not need to be maintained precisely, but must simply be maintained on average.

Fig 4 shows an asymptotic property of $\alpha_{C}$ in a large limit of $\delta^{2}$. The solid line shows the analytical results

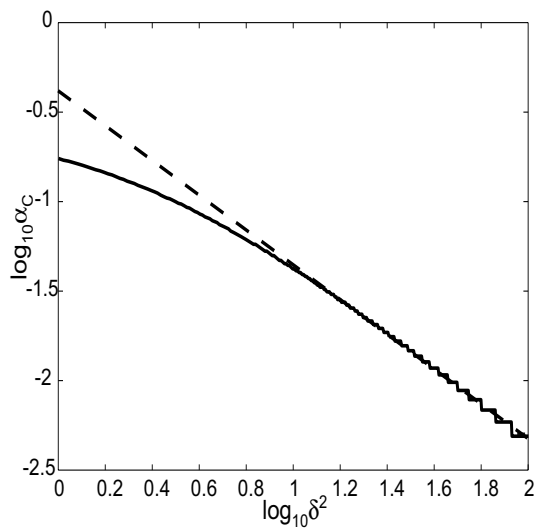

FIG. 4: An asymptotic property of $\alpha_{C}$ in a large limit of $\delta^{2}$ at $f=0.1$ and $\theta=0.52$. The solid line shows the analytical results while the dashed line shows $\log _{10} \alpha_{C}=\log _{10} \frac{2}{\pi \delta^{2}}-$ 1.13. $\alpha_{C}$ converges to 0 as order of $\frac{1}{\delta^{2}}$ with respect to $\delta$, $O\left(\frac{1}{\delta^{2}}\right)$, in the large $\delta^{2}$ limit.

obtained by the same procedure to obtain $\alpha_{C}$ in Fig 2 at $f=0.1$ and $\theta=0.52$ while the dashed line shows $\log _{10} \alpha_{C}=\log _{10} \frac{2}{\pi \delta^{2}}-1.13$. This figure indicates that $\alpha_{C}$ 
converges to 0 as order of $\frac{1}{\delta^{2}}$ with respect to $\delta, O\left(\frac{1}{\delta^{2}}\right)$, in the large $\delta^{2}$ limit 22].

Next, we discuss the dependency of the basin of attraction on $\delta$. Each region between the upper line and the lower line in Fig. 2(a),(b),(c) shows the basin of attraction at $\delta=0.0,1.0,2.0$, respectively. The basin becomes smaller as a value of $\delta$ increases. However, the initial critical overlap $m_{C}$ is unchanged. To introduce a threshold control scheme is known to enlarge the basin of attraction 16, 20, 23].

\section{DISCUSSION}

In this paper, we investigated the impact of the imbalance between LTP and LTD of STDP on the retrieval properties of spatio-temporal patterns, employing an associative memory network. We analytically investigated the retrieval properties using the statistical neurodynamics. When the fluctuation of LTD is assumed to obey the Gaussian distribution with mean 0 and variance $\delta^{2}$, the storage capacity takes a finite value even at large $\delta$. This implies that the balance does not need to be maintained precisely, but must be maintained on average. This mechanism might work in the brain. Furthermore, the storage capacity converges to 0 as order $O\left(\frac{1}{\delta^{2}}\right)$ in the large $\delta^{2}$ limit. Finally, we found that the basin of attraction becomes smaller as the fluctuation of LTD increases while the initial critical overlap remains unchanged.

We found that the storage capacity takes a finite value even at large $\delta$. When $\delta$ is larger than 1.0, LTD sometimes disappears in the learning process. The spatiotemporal patterns seem not to be retrievable. Surprisingly, even in this situation, the patterns are retrievable. This implies that the present model achieves strong robustness against the imbalance between LTP and LTD.

\section{APPENDIX A: DERIVATION OF DYNAMICAL EQUATIONS BY THE STATISTICAL NEURODYNAMICS}

The detail derivation of the dynamical equations 12 15) is given in this appendix. At first, we give a sketch of the derivation. The main point in this derivation is to divide an internal potential $u_{i}(t)$ into two parts, a signal term for a retrieval of a target pattern and a cross-talk noise term that represents contributions from non-target patterns and prevents the target pattern from being retrieved. We evaluate the cross-talk noise term. Specifically, the internal potential $u_{i}(t)$ of $i$-th neuron at time $t$ is expressed as (see equation (9))

$$
\begin{aligned}
u_{i}(t)= & \sum_{\mu=1}^{p}\left(\bar{\xi}_{i}^{\mu+1}-\bar{\xi}_{i}^{\mu-1}\right) m^{\mu}(t) \\
& -\frac{1}{N f(1-f)} \sum_{j=1}^{N} \sum_{\mu=1}^{p} \epsilon_{i j}^{\mu-1} \xi_{i}^{\mu-1} \xi_{j}^{\mu} x_{j}(t)
\end{aligned}
$$

$$
\begin{aligned}
= & \left(\bar{\xi}_{i}^{t+1}-\bar{\xi}_{i}^{t-1}\right) m^{t}(t)+\sum_{\mu \neq t}^{p}\left(\bar{\xi}_{i}^{\mu+1}-\bar{\xi}_{i}^{\mu-1}\right) m^{\mu}(t) \\
& -\frac{1}{N f(1-f)} \sum_{j=1}^{N} \sum_{\mu=1}^{p} \epsilon_{i j}^{\mu-1} \xi_{i}^{\mu-1} \xi_{j}^{\mu} x_{j}(t) \\
= & \left(\bar{\xi}_{i}^{t+1}-\bar{\xi}_{i}^{t-1}\right) m^{t}(t)+z_{i}(t),
\end{aligned}
$$

where $\bar{\xi}_{i}^{\mu}=\xi_{i}^{\mu}-f, m^{\mu}(t)$ is the overlap between $\boldsymbol{\xi}^{\mu}(t)$ and $\boldsymbol{x}(t)$ and is given by

$$
m^{\mu}(t)=\frac{1}{N f(1-f)} \sum_{i=1}^{N} \bar{\xi}_{i}^{\mu} x_{i}(t),
$$

and

$$
\begin{aligned}
z_{i}(t)= & \sum_{\mu \neq t}^{p}\left(\bar{\xi}_{i}^{\mu+1}-\bar{\xi}_{i}^{\mu-1}\right) m^{\mu}(t) \\
& -\frac{1}{N f(1-f)} \sum_{j=1}^{N} \sum_{\mu=1}^{p} \epsilon_{i j}^{\mu-1} \xi_{i}^{\mu-1} \xi_{j}^{\mu} x_{j}(t) .
\end{aligned}
$$

The first term in equation A3 is the signal term and the second term is the cross-talk noise term. Since $x_{i}(t)$ in equation (A4) depends on $\xi_{i}^{\mu}$, the distribution of the cross-talk noise term $z_{i}(t)$ is unknown. The dependence on $\xi_{i}^{\mu}$ is extracted from $x_{i}(t)$ using the Taylor expansion (see equation (A10). In the thermodynamic limit, $N \rightarrow$ $\infty, m^{\mu}(t)$ tends to be deterministic. Therefore, $x_{i}^{\{\mu\}}(t)$, which denotes that it does not include $\xi_{i}^{\mu}$, is independent of $\xi_{i}^{\mu}$. This enables us to assume that the cross-talk noise term $z_{i}(t)$ obeys a Gaussian distribution with mean 0 and variance $\sigma^{2}[16,20]$. Since the distribution of the crosstalk noise term is known, the recursive equation of the overlap is obtained (see equation (A35)).

To extract the dependence on $\xi_{i}^{\mu}$ from $x_{i}(t)$, the state of the $i$-th neuron at time $t+1$ is transformed:

$$
\begin{aligned}
x_{i}(t+1)= & F\left(u_{i}(t)-\theta\right) \\
= & F\left(\sum_{\nu=1}^{p}\left(\bar{\xi}_{i}^{\nu+1}-\bar{\xi}_{i}^{\nu-1}\right) m^{\nu}(t)\right. \\
& \left.-\frac{1}{N f(1-f)} \sum_{j=1}^{N} \sum_{\nu=1}^{p} \epsilon_{i j}^{\nu-1} \xi_{i}^{\nu-1} \xi_{j}^{\nu} x_{j}(t)-\theta\right) .
\end{aligned}
$$

The first term in the function $F(\cdot)$ of equation (A6) is transformed using the periodic boundary condition of $\xi_{i}^{p+1}=\xi_{i}^{1}$ and $\xi_{i}^{0}=\xi_{i}^{p}$.

$$
\begin{aligned}
& \sum_{\nu=1}^{p}\left(\bar{\xi}_{i}^{\nu+1}-\bar{\xi}_{i}^{\nu-1}\right) m^{\nu}(t) \\
= & \sum_{\nu=1}^{p} \bar{\xi}_{i}^{\nu+1} m^{\nu}(t)-\sum_{\nu=1}^{p} \bar{\xi}_{i}^{\nu-1} m^{\nu}(t) \\
= & \sum_{\nu^{\prime}=1}^{p} \bar{\xi}_{i}^{\nu^{\prime}} m^{\nu^{\prime}-1}(t)-\sum_{\nu^{\prime}=1}^{p} \bar{\xi}_{i}^{\nu^{\prime}} m^{\nu+1}(t)
\end{aligned}
$$




$$
=\sum_{\nu=1}^{p} \bar{\xi}_{i}^{\nu}\left\{m^{\nu-1}(t)-m^{\nu+1}(t)\right\}
$$

The second term in the function $F(\cdot)$ of equation (A6) is transformed using the periodic boundary condition:

$$
\begin{aligned}
& \frac{1}{N f(1-f)} \sum_{j=1}^{N} \sum_{\nu=1}^{p} \epsilon_{i j}^{\nu-1} \xi_{i}^{\nu-1} \xi_{j}^{\nu} x_{j}(t) \\
= & \frac{1}{N f(1-f)} \sum_{j=1}^{N} \sum_{\nu^{\prime}=1}^{p} \epsilon_{i j}^{\nu^{\prime}} \xi_{i}^{\nu^{\prime}} \xi_{j}^{\nu^{\prime}+1} x_{j}(t) .
\end{aligned}
$$

To extract the dependency on $\xi_{i}^{\mu}$ from $x_{i}(t+1)$, using the equation (A7) and (A8), $x(t+1)$ is divided into two parts, the terms which include $\xi_{i}^{\mu}$ and the terms which do not include $\xi_{i}^{\mu}$ :

$$
\begin{aligned}
x_{i}(t+1)= & F\left(\sum_{\nu=1}^{p} \bar{\xi}_{i}^{\nu}\left\{m^{\nu-1}(t)-m^{\nu+1}(t)\right\}\right. \\
& \left.-\frac{1}{N f(1-f)} \sum_{j=1}^{N} \sum_{\nu=1}^{p} \epsilon_{i j}^{\nu} \xi_{i}^{\nu} \xi_{j}^{\nu+1} x_{j}(t)-\theta\right) \\
= & F\left(\bar{\xi}_{i}^{\mu}\left\{m^{\mu-1}(t)-m^{\mu+1}(t)\right\}\right. \\
& -\frac{1}{N f(1-f)} \sum_{j=1}^{N} \epsilon_{i j}^{\mu} \xi_{i}^{\mu} \xi_{j}^{\mu+1} x_{j}(t) \\
& +\sum_{\nu \neq \mu}^{p} \bar{\xi}_{i}^{\nu}\left\{m^{\nu-1}(t)-m^{\nu+1}(t)\right\} \\
& \left.-\frac{1}{N f(1-f)} \sum_{j=1}^{N} \sum_{\nu \neq \mu}^{p} \epsilon_{i j}^{\nu} \xi_{i}^{\nu} \xi_{j}^{\nu+1} x_{j}(t)-\theta\right) .
\end{aligned}
$$

At time $t$, the pattern $\boldsymbol{\xi}^{t}$ is designed to be retrieved. Therefore, we can assume that $m^{t}(t)$ is order of 1 with respect to $N, m^{t}(t) \sim O(1)$, and $m^{\mu}(t)(\mu \neq t)$ is order of $1 / \sqrt{N}$ with respect to $N, m^{\mu}(t) \sim O(1 / \sqrt{N})$. Since $m^{\mu}(t) \sim O(1 / \sqrt{N}), m^{\mu-1}(t)$ and $m^{\mu+1}(t)$ are order of $1 / \sqrt{N}$ with respect to $N$. Since $m^{\mu+1}(t) \sim O(1 / \sqrt{N})$ and $\epsilon_{i j}^{\mu} \sim O(1)$, the second term in equation (A9) can be considered to be order of $1 / \sqrt{N}$ with respect to $N$. In the thermodynamic limit, $N \rightarrow \infty$, the first and the second terms in equation (A9) are small. Using the Taylor expansion up to the first order of $x_{i}(t+1), x_{i}(t+1)$ is transformed:

$$
x_{i}(t+1)=x_{i}^{(\mu)}(t+1)+u_{i}^{\{\mu\}}(t) x_{i}^{\prime(\mu)}(t+1),
$$

where $x_{i}^{(\mu)}(t+1)$ is independent of $\xi_{i}^{\mu}, x_{i}^{\prime(\mu)}(t+1)$ is differential of $x_{i}^{(\mu)}(t+1)$, and

$$
\begin{aligned}
u_{i}^{\{\mu\}}(t) & =\bar{\xi}_{i}^{\mu}\left\{m^{\mu-1}(t)-m^{\mu+1}(t)\right\} \\
& -\frac{1}{N f(1-f)} \sum_{j=1}^{N} \epsilon_{i j}^{\mu} \xi_{i}^{\mu} \xi_{j}^{\mu+1} x_{j}(t), \\
x_{i}^{(\mu)}(t+1) & =F\left(u_{i}(t)-u_{i}^{\{\mu\}}(t)-\theta\right), \\
x_{i}^{\prime(\mu)}(t+1) & =F^{\prime}\left(u_{i}(t)-u_{i}^{\{\mu\}}(t)-\theta\right) .
\end{aligned}
$$

We assume that the function $F(\cdot)$ is differentiable. This assumption is valid since the average of $F(\cdot)$ over a Gaussian noise term will be taken in a later step (see equation (A34)). For $\mu \neq t$, the overlap $m^{\mu}(t)$ is expressed as

$$
\begin{aligned}
m^{\mu}(t)= & \frac{1}{N f(1-f)} \sum_{i=1}^{N}\left(\xi_{i}^{\mu}-f\right) x_{i}(t)=\frac{1}{N f(1-f)} \sum_{i=1}^{N} \bar{\xi}_{i}^{\mu}\left\{x_{i}^{(\mu)}(t)+u_{i}^{\{\mu\}}(t-1) x_{i}^{\prime(\mu)}(t)\right\} \\
= & \frac{1}{N f(1-f)} \sum_{i=1}^{N} \bar{\xi}_{i}^{\mu} x_{i}^{(\mu)}(t)+\frac{1}{N f(1-f)} \sum_{i=1}^{N}\left(\bar{\xi}_{i}^{\mu}\right)^{2}\left\{m^{\mu-1}(t-1)-m^{\mu+1}(t-1)\right\} x_{i}^{\prime(\mu)}(t) \\
& -\left(\frac{1}{N f(1-f)}\right)^{2} \sum_{i=1}^{N} \bar{\xi}_{i}^{\mu} \sum_{j=1}^{N} \epsilon_{i j}^{\mu} \xi_{i}^{\mu} \xi_{j}^{\mu+1} x_{j}(t-1) x_{i}^{\prime(\mu)}(t) .
\end{aligned}
$$

If the averages over $\xi_{j}^{\mu}(\mu \neq t)$ and $\epsilon_{j i}^{\mu}(\mu \neq t)$ are taken in the right-hand side of equation A15, the third term vanishes since $\mathrm{E}\left[\epsilon_{j i}^{\mu}\right]=0$. Since the third term including $x_{j}(t-1)$ depends on both $\xi_{j}^{\mu}$ and $\epsilon_{j i}^{\mu}, \xi_{j}^{\mu}$ and $\epsilon_{j i}^{\mu}$ are extracted from $x_{j}(t)$ before the averages are taken:

$$
\begin{aligned}
x_{j}(t+1)= & F\left(\bar{\xi}_{j}^{\mu}\left\{m^{\mu-1}(t)-m^{\mu+1}(t)\right\}-\frac{1}{N f(1-f)} \sum_{k \neq i}^{N} \epsilon_{j k}^{\mu} \xi_{j}^{\mu} \xi_{k}^{\mu+1} x_{k}(t)-\frac{1}{N f(1-f)} \sum_{\nu \neq \mu}^{p} \epsilon_{j i}^{\nu} \xi_{j}^{\nu} \xi_{i}^{\nu+1} x_{i}(t)\right. \\
& \left.-\frac{1}{N f(1-f)} \epsilon_{j i}^{\mu} \xi_{j}^{\mu} \xi_{i}^{\mu+1} x_{i}(t)+\sum_{\nu \neq \mu}^{p} \bar{\xi}_{j}^{\nu}\left\{m^{\nu-1}(t)-m^{\nu+1}(t)\right\}+\frac{1}{N f(1-f)} \sum_{k \neq i}^{N} \sum_{\nu \neq \mu}^{p} \epsilon_{j k}^{\nu} \xi_{j}^{\nu} \xi_{k}^{\nu+1} x_{k}(t)-\theta\right)
\end{aligned}
$$




$$
=x_{j}^{(\mu)\left(\epsilon_{j i}^{\mu}\right)}(t+1)+u_{j}^{\left\{\mu, \epsilon_{j i}^{\mu}\right\}}(t) x_{j}^{(\mu)\left(\epsilon_{j i}^{\mu}\right)}(t+1),
$$

where $x_{j}^{(\mu)\left(\epsilon_{j i}^{\mu}\right)}(t)$, which is independent of both $\xi_{j}^{\mu}(t)$ and $\epsilon_{j i}^{\mu}$, is the differential of $x_{j}^{(\mu)\left(\epsilon_{j i}^{\mu}\right)}(t)$ and

$$
\begin{aligned}
u_{j}^{\left\{\mu, \epsilon_{j i}^{\mu}\right\}}(t)= & \bar{\xi}_{j}^{\mu}\left\{m^{\mu-1}(t)-m^{\mu+1}(t)\right\}-\frac{1}{N f(1-f)} \sum_{k \neq i}^{N} \epsilon_{j k}^{\mu} \xi_{j}^{\mu} \xi_{k}^{\mu+1} x_{k}(t)-\frac{1}{N f(1-f)} \sum_{\nu \neq \mu}^{p} \epsilon_{j i}^{\nu} \xi_{j}^{\nu} \xi_{i}^{\nu+1} x_{i}(t) \\
& -\frac{1}{N f(1-f)} \epsilon_{j i}^{\mu} \xi_{j}^{\mu} \xi_{i}^{\mu+1} x_{i}(t),
\end{aligned}
$$$$
x_{j}^{(\mu)\left(\epsilon_{j i}^{\mu}\right)}(t+1)=F\left(u_{j}(t)-u_{j}^{\left\{\mu, \epsilon_{j i}^{\mu}\right\}}(t)-\theta\right),(\mathrm{A} 1
$$$$
x_{j}^{\prime(\mu)\left(\epsilon_{j i}^{\mu}\right)}(t+1)=F^{\prime}\left(u_{j}(t)-u_{j}^{\left\{\mu, \epsilon_{j i}^{\mu}\right\}}(t)-\theta\right) \text {. }
$$

$$
+U(t)\left\{m^{\mu-1}(t-1)-m^{\mu+1}(t-1)\right\},
$$

The first, second and third term in the right-hand side of equation (A18) is order of $1 / \sqrt{N}$ with respect to $N$ and the fourth term is order of $1 / N$ with respect to $N$. In the thermodynamic limit, $N \rightarrow \infty, u_{j}^{\left\{\mu, \epsilon_{j i}^{\mu}\right\}}(t)$ is small and equation (A16) equals equation (A17). Substituting equation (A17) into the right-hand side of equation A15 and averaging the resultant expressions over $\xi_{j}^{\mu}$ and $\epsilon_{j i}^{\mu}$ and using $\mathrm{E}\left[\epsilon_{j i}^{\mu}\right]=0$ yield to the following equation for $m^{\mu}(t)(\mu \neq t)$ :

$$
\begin{aligned}
m^{\mu}(t)= & \frac{1}{N f(1-f)} \sum_{i=1}^{N} \bar{\xi}_{i}^{\mu} x_{i}^{(\mu)}(t) \\
& +\frac{1}{N f(1-f)} \sum_{i=1}^{N}\left(\bar{\xi}_{i}^{\mu}\right)^{2}\left\{m^{\mu-1}(t-1)\right. \\
& \left.-m^{\mu+1}(t-1)\right\} x_{i}^{(\mu)}(t) \\
= & \frac{1}{N f(1-f)} \sum_{i=1}^{N} \bar{\xi}_{i}^{\mu} x_{i}^{(\mu)}(t)
\end{aligned}
$$

where $U(t)=\frac{1}{N} \sum_{i=1}^{N} x_{i}^{\prime(\mu)}(t)$. Substituting equation (A22) into equation (Â2) yields

$$
\begin{aligned}
u_{i}(t)= & \left(\bar{\xi}_{i}^{t+1}-\bar{\xi}_{i}^{t-1}\right) m^{t}(t) \\
& +\frac{1}{N f(1-f)} \sum_{j=1}^{N} \sum_{\mu \neq t}^{p}\left(\bar{\xi}_{i}^{\mu+1}-\bar{\xi}_{i}^{\mu-1}\right) \bar{\xi}_{j}^{\mu} x_{j}^{(\mu)}(t) \\
& +\sum_{\mu \neq t}^{p} U(t)\left\{\bar{\xi}_{i}^{\mu+1} m^{\mu-1}(t-1)\right. \\
& \left.-2 \bar{\xi}_{i}^{\mu-1} m^{\mu-1}(t-1)+\bar{\xi}_{i}^{\mu-1} m^{\mu+1}(t-1)\right\} \\
& \left.-\frac{1}{N f(1-f)} \sum_{j=1}^{N} \sum_{\mu=1}^{p} \epsilon_{i j}^{\mu-1} \xi_{i}^{\mu-1} \xi_{j}^{\mu} x_{j}(t) . \quad \text { (A } 23\right)
\end{aligned}
$$

Substituting equation (A17) into the last term of equation A23 yields the following expression for $u_{i}(t)$ :

$$
\begin{aligned}
u_{i}(t)= & \left(\bar{\xi}_{i}^{t+1}-\bar{\xi}_{i}^{t-1}\right) m^{t}(t)+\frac{1}{N f(1-f)} \sum_{j=1}^{N} \sum_{\mu \neq t}^{p}\left(\bar{\xi}_{i}^{\mu+1}-\bar{\xi}_{i}^{\mu-1}\right) \bar{\xi}_{j}^{\mu} x_{j}^{(\mu)}(t) \\
& +\sum_{\mu \neq t}^{p} U(t)\left\{\bar{\xi}_{i}^{\mu+1} m^{\mu-1}(t-1)-2 \bar{\xi}_{i}^{\mu-1} m^{\mu-1}(t-1)+\bar{\xi}_{i}^{\mu-1} m^{\mu+1}(t-1)\right\} \\
& -\frac{1}{N f(1-f)} \sum_{j=1}^{N} \sum_{\mu=1}^{p} \epsilon_{i j}^{\mu-1} \xi_{i}^{\mu-1} \xi_{j}^{\mu}\left\{x_{j}^{(\mu)\left(\epsilon_{j i}^{\mu}\right)}(t)+u_{j}^{\left\{\mu, \epsilon_{j i}^{\mu}\right\}}(t-1) x_{j}^{\prime(\mu)\left(\epsilon_{j i}^{\mu}\right)}(t)\right\} \\
= & \left(\bar{\xi}_{i}^{t+1}-\bar{\xi}_{i}^{t-1}\right) m^{t}(t)+\frac{1}{N f(1-f)} \sum_{j=1}^{N} \sum_{\mu \neq t}^{p}\left(\bar{\xi}_{i}^{\mu+1}-\bar{\xi}_{i}^{\mu-1}\right) \bar{\xi}_{j}^{\mu} x_{j}^{(\mu)}(t) \\
& +\sum_{\mu \neq t}^{p} U(t)\left\{\bar{\xi}_{i}^{\mu+1} m^{\mu-1}(t-1)-2 \bar{\xi}_{i}^{\mu-1} m^{\mu-1}(t-1)+\bar{\xi}_{i}^{\mu-1} m^{\mu+1}(t-1)\right\} \\
& -\frac{1}{N f(1-f)} \sum_{j=1}^{N} \sum_{\mu=1}^{p} \epsilon_{i j}^{\mu-1} \xi_{i}^{\mu-1} \xi_{j}^{\mu} x_{j}^{(\mu)\left(\epsilon_{j i}^{\mu}\right)}(t)
\end{aligned}
$$




$$
\begin{aligned}
& -\frac{1}{N f(1-f)} \sum_{j=1}^{N} \sum_{\mu=1}^{p} \epsilon_{i j}^{\mu-1} \xi_{i}^{\mu-1} \xi_{j}^{\mu} \bar{\xi}_{j}^{\mu}\left\{m^{\mu-1}(t-1)-m^{\mu+1}(t-1)\right\} x_{j}^{(\mu)\left(\epsilon_{j i}^{\mu}\right)}(t) \\
& +\left(\frac{1}{N f(1-f)}\right)^{2} \sum_{j=1}^{N} \sum_{\mu=1}^{p} \epsilon_{i j}^{\mu-1} \xi_{i}^{\mu-1} \xi_{j}^{\mu} \sum_{k \neq i}^{N} \epsilon_{j k}^{\mu} \xi_{j}^{\mu} \xi_{k}^{\mu+1} x_{k}(t-1) x_{j}^{\prime(\mu)\left(\epsilon_{j i}^{\mu}\right)}(t) \\
& +\left(\frac{1}{N f(1-f)}\right)^{2} \sum_{j=1}^{N} \sum_{\mu=1}^{p} \epsilon_{i j}^{\mu-1} \xi_{i}^{\mu-1} \xi_{j}^{\mu} \sum_{\nu \neq \mu}^{p} \epsilon_{j i}^{\nu} \xi_{j}^{\nu} \xi_{i}^{\nu+1} x_{i}(t-1) x_{j}^{(\mu)\left(\epsilon_{j i}^{\mu}\right)}(t) \\
& +\left(\frac{1}{N f(1-f)}\right)^{2} \sum_{j=1}^{N} \sum_{\mu=1}^{p} \epsilon_{i j}^{\mu-1} \xi_{i}^{\mu-1} \xi_{j}^{\mu} \epsilon_{j i}^{\mu} \xi_{j}^{\mu} \xi_{i}^{\mu+1} x_{i}(t-1) x_{j}^{(\mu)\left(\epsilon_{j i}^{\mu}\right)}(t) .
\end{aligned}
$$

The fifth, sixth, seventh and eighth terms vanish since $\mathrm{E}\left[\epsilon_{i j}^{\mu}\right]=0$, and this yields

$$
\begin{aligned}
u_{i}(t)= & \left(\bar{\xi}_{i}^{t+1}-\bar{\xi}_{i}^{t-1}\right) m^{t}(t)+\frac{1}{N f(1-f)} \sum_{j=1}^{N} \sum_{\mu \neq t}^{p}\left(\bar{\xi}_{i}^{\mu+1}-\bar{\xi}_{i}^{\mu-1}\right) \bar{\xi}_{j}^{\mu} x_{j}^{(\mu)}(t) \\
& +\sum_{\mu \neq t}^{p} U(t)\left\{\bar{\xi}_{i}^{\mu+1} m^{\mu-1}(t-1)-2 \bar{\xi}_{i}^{\mu-1} m^{\mu-1}(t-1)+\bar{\xi}_{i}^{\mu-1} m^{\mu+1}(t-1)\right\} \\
& -\frac{1}{N f(1-f)} \sum_{j=1}^{N} \sum_{\mu=1}^{p} \epsilon_{i j}^{\mu-1} \xi_{i}^{\mu-1} \xi_{j}^{\mu} x_{j}^{(\mu)\left(\epsilon_{j i}^{\mu}\right)}(t) \\
= & \left(\bar{\xi}_{i}^{t+1}-\bar{\xi}_{i}^{t-1}\right) m^{t}(t)+z_{i}(t),
\end{aligned}
$$

where

$$
\begin{aligned}
z_{i}(t)= & \frac{1}{N f(1-f)} \sum_{j=1}^{N} \sum_{\mu \neq t}^{p}\left(\bar{\xi}_{i}^{\mu+1}-\bar{\xi}_{i}^{\mu-1}\right) \bar{\xi}_{j}^{\mu} x_{j}^{(\mu)}(t)+\sum_{\mu \neq t}^{p} U(t)\left\{\bar{\xi}_{i}^{\mu+1} m^{\mu-1}(t-1)-2 \bar{\xi}_{i}^{\mu-1} m^{\mu-1}(t-1)\right. \\
& \left.+\bar{\xi}_{i}^{\mu-1} m^{\mu+1}(t-1)\right\}-\frac{1}{N f(1-f)} \sum_{j=1}^{N} \sum_{\mu=1}^{p} \epsilon_{i j}^{\mu-1} \xi_{i}^{\mu-1} \xi_{j}^{\mu} x_{j}^{(\mu)\left(\epsilon_{j i}^{\mu}\right)}(t) .
\end{aligned}
$$

$z_{i}(t)$ is the cross-talk noise term. We assume that the cross-talk noise obeys a Gaussian distribution with mean 0 and time-dependent variance $\sigma^{2}(t): \mathrm{E}\left[z_{i}(t)\right]=0, \mathrm{E}\left[\left(z_{i}(t)\right)^{2}\right]=\sigma^{2}(t)\left[16\right.$, 20]. The first and second term of $z_{i}(t)$ are the same cross-talk noise term as that of our previous work [16]. The square of $z_{i}(t)$ is given by

$$
\begin{aligned}
\left\{z_{i}(t)\right\}^{2}= & \left(\frac{1}{N f(1-f)}\right)^{2} \sum_{j=1}^{N} \sum_{\mu \neq t}^{p}\left(\bar{\xi}_{i}^{\mu+1}-\bar{\xi}_{i}^{\mu-1}\right)^{2}\left(\bar{\xi}_{j}^{\mu}\right)^{2}\left\{x_{j}^{(\mu)}(t)\right\}^{2} \\
& +\sum_{\nu \neq t}^{p} U(t)^{2}\left\{\bar{\xi}_{i}^{\nu+1} m^{\nu-1}(t-1)-2 \bar{\xi}_{i}^{\nu-1} m^{\nu-1}(t-1)+\bar{\xi}_{i}^{\nu-1} m^{\nu+1}(t-1)\right\}^{2} \\
& +\left(\frac{1}{N f(1-f)}\right)^{2} \sum_{j=1}^{N} \sum_{\mu=1}^{p}\left(\epsilon_{i j}^{\mu-1}\right)^{2}\left(\xi_{i}^{\mu-1}\right)^{2}\left(\xi_{j}^{\mu}\right)^{2}\left\{x_{j}^{(\mu)\left(\epsilon_{j i}^{\mu}\right)}(t)\right\}^{2} \\
& +\frac{2}{N f(1-f)} \sum_{j=1}^{N} \sum_{\mu \neq t}^{p}\left(\bar{\xi}_{i}^{\mu+1}-\bar{\xi}_{i}^{\mu-1}\right) \bar{\xi}_{j}^{\mu} x_{j}^{(\mu)}(t) \\
& \times \sum_{\mu^{\prime} \neq t}^{p} U(t)\left\{\bar{\xi}_{i}^{\mu^{\prime}+1} m^{\mu^{\prime}-1}(t-1)-2 \bar{\xi}_{i}^{\mu^{\prime}-1} m^{\mu^{\prime}-1}(t-1)+\bar{\xi}_{i}^{\mu^{\prime}-1} m^{\mu^{\prime}+1}(t-1)\right\} \\
& +2 \sum_{\mu \neq t}^{p} U(t)\left\{\bar{\xi}_{i}^{\mu+1} m^{\mu-1}(t-1)-2 \bar{\xi}_{i}^{\mu-1} m^{\mu-1}(t-1)+\bar{\xi}_{i}^{\mu-1} m^{\mu+1}(t-1)\right\} \\
& \times \frac{1}{N f(1-f)} \sum_{j=1}^{N} \sum_{\mu=1}^{p} \epsilon_{i j}^{\mu-1} \xi_{i}^{\mu-1} \xi_{j}^{\mu} x_{j}^{(\mu)\left(\epsilon_{j i}^{\mu}\right)}(t)
\end{aligned}
$$




$$
\begin{aligned}
& +2\left(\frac{1}{N f(1-f)}\right)^{2} \sum_{j=1}^{N} \sum_{\mu^{\prime}=1}^{p} \epsilon_{i j}^{\mu^{\prime}-1} \xi_{i}^{\mu^{\prime}-1} \xi_{j}^{\mu^{\prime}} x_{j}^{\left(\mu^{\prime}\right)\left(\epsilon_{j i}^{\mu^{\prime}}\right)}(t) \sum_{k=1}^{N} \sum_{\mu^{\prime} \neq t}^{p}\left(\bar{\xi}_{i}^{\mu^{\prime}+1}-\bar{\xi}_{i}^{\mu^{\prime}-1}\right) \bar{\xi}_{k}^{\mu^{\prime}} x_{k}^{\left(\mu^{\prime}\right)}(t) \\
= & \left(\frac{1}{N f(1-f)}\right)^{2} \sum_{j=1}^{N} \sum_{\mu \neq t}^{p}\left(\bar{\xi}_{i}^{\mu+1}-\bar{\xi}_{i}^{\mu-1}\right)^{2}\left(\bar{\xi}_{j}^{\mu}\right)^{2}\left\{x_{j}^{(\mu)}(t)\right\}^{2} \\
& +\sum_{\nu \neq t}^{p} U(t)^{2}\left\{\bar{\xi}_{i}^{\nu+1} m^{\nu-1}(t-1)-2 \bar{\xi}_{i}^{\nu-1} m^{\nu-1}(t-1)+\bar{\xi}_{i}^{\nu-1} m^{\nu+1}(t-1)\right\}^{2} \\
& +\left(\frac{1}{N f(1-f)}\right)^{2} \sum_{j=1}^{N} \sum_{\mu=1}^{p}\left(\epsilon_{i j}^{\mu-1}\right)^{2}\left(\xi_{i}^{\mu-1}\right)^{2}\left(\xi_{j}^{\mu}\right)^{2}\left\{x_{j}^{(\mu)\left(\epsilon_{j i}^{\mu}\right)}(t)\right\}^{2} \\
= & \sum_{a=0}^{t} 2(a+1) \mathrm{C}_{(a+1)} \alpha q(t-a) \prod_{b=1}^{a} U^{2}(t-b+1)+\frac{\alpha \delta^{2}}{(1-f)^{2}} q(t),
\end{aligned}
$$

where $p=\alpha N, q(t)=\frac{1}{N} \sum_{i=1}^{N}\left\{x_{i}^{(\mu)}(t)\right\}^{2},{ }_{b} \mathrm{C}_{a}=$ $\frac{b !}{a !(b-a) !}, a !$ is the factorial with positive integer $a$ and $\mathrm{E}\left[\left(\epsilon_{i j}^{\mu}\right)^{2}\right]=\delta^{2}$. Since $\mathrm{E}\left[\epsilon_{i j}^{\mu}\right]=0$, the fourth, fifth and sixth terms in equation (A29) vanish. We applied the relationship $\sum_{a=0}^{b}\left({ }_{b} \mathrm{C}_{a}\right)^{2}={ }_{2 b} \mathrm{C}_{b}$ in this derivation. Since $\mathrm{E}\left[z_{i}(t)\right]=0$, the variance $\sigma^{2}(t)$ is equal to $\mathrm{E}\left[\left\{z_{i}(t)\right\}^{2}\right]$. We then get the recursive equation for $\sigma^{2}(t)$ :

$$
\begin{aligned}
\sigma^{2}(t)= & \sum_{a=0}^{t} 2(a+1) \mathrm{C}_{(a+1)} \alpha q(t-a) \prod_{b=1}^{a} U^{2}(t-b+1) \\
& +\frac{\alpha \delta^{2}}{(1-f)^{2}} q(t) .
\end{aligned}
$$

The overlap between the state $\boldsymbol{x}(t)$ and the retrieval pattern $\boldsymbol{\xi}^{t}$ is given by

$$
\begin{aligned}
m^{t}(t)= & \frac{1}{N f(1-f)} \sum_{i=1}^{N}\left(\xi_{i}^{t}-f\right) x_{i}(t) \\
= & \frac{1}{N f(1-f)} \sum_{i=1}^{N}\left(\xi_{i}^{t}-f\right) \\
& \times F\left(\left(\xi_{i}^{t}-\xi_{i}^{t-2}\right) m^{t-1}(t-1)+z_{i}(t-1)-\theta\right) .
\end{aligned}
$$

Since $u_{i}(t)$ is independent and identical distribution (i.i.d.), by the law of large numbers, the average over $i$ can be replaced by an average over the memory patterns $\xi^{\mu}$ and the Gaussian noise term $z \sim \mathcal{N}\left(0, \sigma^{2}\right)$. Then, the recursive equation for the overlap $m^{t}(t)$ is transformed:

$$
\begin{aligned}
m^{t}(t)= & \frac{1}{f(1-f)} \frac{1}{\sqrt{2 \pi} \sigma} \int_{-\infty}^{\infty} d z e^{-\frac{z^{2}}{2 \sigma^{2}}}\left\langle\left\langle\left(\xi^{t}-f\right)\right.\right. \\
& \left.\left.\times F\left(\left(\xi^{t}-\xi^{t-2}\right) m^{t-1}(t-1)+z-\theta\right)\right\rangle\right\rangle \\
= & \frac{1}{f(1-f)} \frac{1}{\sqrt{2 \pi}} \int_{-\infty}^{\infty} d \tilde{z} e^{-\frac{\tilde{z}^{2}}{2}}\left\langle\left\langle\left(\xi^{t}-f\right)\right.\right.
\end{aligned}
$$

$$
\begin{array}{r}
\left.\left.\times F\left(\left(\xi^{t}-\xi^{t-2}\right) m^{t-1}(t-1)+\sigma(t-1) \tilde{z}-\theta\right)\right\rangle\right\rangle \\
(\mathrm{A} 34) \\
=\frac{1-2 f}{2} \operatorname{erf}\left(\phi_{0}\right)-\frac{1-f}{2} \operatorname{erf}\left(\phi_{1}\right)+\frac{f}{2} \operatorname{erf}\left(\phi_{2}\right),(\mathrm{A} 35)
\end{array}
$$

where $\tilde{z}=z / \sigma, \operatorname{erf}(y)=\frac{2}{\sqrt{\pi}} \int_{0}^{y} \exp \left(-u^{2}\right) d u$, and $\phi_{0}=$ $\frac{\theta}{\sqrt{2} \sigma(t-1)}, \phi_{1}=\frac{-m^{t-1}(t-1)+\theta}{\sqrt{2} \sigma(t-1)}, \phi_{2}=\frac{m^{t-1}(t-1)+\theta}{\sqrt{2} \sigma(t-1)}$, and $\langle\langle\cdot\rangle\rangle$ denotes an average over the memory pattern $\xi^{\mu}$. Since $x_{i}^{\prime}(t)-x_{i}^{\prime(\mu)}(t) \sim O\left(\frac{1}{\sqrt{N}}\right)$ and the thermodynamic limit, $N \rightarrow \infty$, is considered, $x_{i}^{\prime(\mu)}(t)=x_{i}^{\prime}(t)$. Using this relationship, we derive $U(t)$ :

$$
\begin{aligned}
U(t)= & \frac{1}{N} \sum_{i=1}^{N} x_{i}^{\prime(\mu)}(t)=\frac{1}{N} \sum_{i=1}^{N} x_{i}^{\prime}(t) \\
= & \frac{1}{f(1-f)} \frac{1}{\sqrt{2 \pi}} \int_{-\infty}^{\infty} d z e^{-\frac{z^{2}}{2}} \\
& \times\left\langle\left\langleF ^ { \prime } \left(\left(\xi^{t}-\xi^{t-2}\right) m^{t-1}(t-1)\right.\right.\right. \\
& +\sigma(t-1) z-\theta)\rangle\rangle \\
= & \frac{1}{f(1-f)} \frac{1}{\sqrt{2 \pi}} \int_{-\infty}^{\infty} d z e^{-\frac{z^{2}}{2}} z \\
& \times\left\langle\left\langleF \left(\left(\xi^{t}-\xi^{t-2}\right) m^{t-1}(t-1)\right.\right.\right. \\
& +\sigma(t-1) z-\theta)\rangle\rangle \\
= & \frac{1}{\sqrt{2 \pi} \sigma(t-1)}\left\{\left(1-2 f+2 f^{2}\right) e^{-\phi_{0}^{2}}\right. \\
& \left.+f(1-f)\left(e^{-\phi_{1}^{2}}+e^{-\phi_{2}^{2}}\right)\right\} .
\end{aligned}
$$

Since $x_{i}(t)-x_{i}^{(\mu)}(t) \sim O\left(\frac{1}{\sqrt{N}}\right)$ and $N \rightarrow \infty, x_{i}^{(\mu)}(t)=$ $x_{i}(t)$. Using this relationship, we derive $q(t)$ :

$$
q(t)=\frac{1}{N} \sum_{i=1}^{N}\left\{x_{i}^{(\mu)}(t)\right\}^{2}=\frac{1}{N} \sum_{i=1}^{N}\left\{x_{i}(t)\right\}^{2}
$$




$$
\begin{aligned}
= & \frac{1}{f(1-f)} \frac{1}{\sqrt{2 \pi}} \int_{-\infty}^{\infty} d z e^{-\frac{z^{2}}{2}} \\
& \times\left\langle\left\langleF ^ { 2 } \left(\left(\xi^{t}-\xi^{t-2}\right) m^{t-1}(t-1)\right.\right.\right. \\
& +\sigma(t-1) z-\theta)\rangle\rangle
\end{aligned}
$$

[1] G. Q. Bi and M. M. Poo, Journal of Neuroscience 18, 10464 (1998).

[2] H. Markram, J. Lübke, M. Frotscher, and B. Sakmann, Science 275, 213 (1997).

[3] L. I. Zhang, H. W. Tao, C. E. Holt, W. A. Harris, and M. M. Poo, Nature 395, 37 (1998).

[4] S. Song, K. D. Miller, and L. F. Abbott, Nature Neuroscience 3, 919 (2000).

[5] L. F. Abbott and S. Song, in Advances in Neural Information Processing Systems, edited by M. S. Kearns, S. Solla, and D. Cohn (Cambridge, MA:MIT Press, 1999), vol. 11, pp. 69-75.

[6] J. Rubin, D. D. Lee, and H. Sompolinsky, Physical Review Letters 86, 364 (2001).

[7] N. Levy, D. Horn, I. Meilijson, and E. Ruppin, Nerural Networks 14, 815 (2001).

[8] M. C. W. van Rossum, G. Q. Bi, and G. G. Turrigiano, Journal of Neuroscience 20, 8812 (2001).

[9] S. Song and L. F. Abbott, Neuron 32, 339 (2001).

[10] W. Gerstner, R. Kempter, J. L. van Hemmen, and H. Wagner, Nature 383, 76 (1996).

[11] R. Kempter, W. Gerstner, and J. L. van Hemmen, Physical Review E 59, 4498 (1999).

[12] W. M. Kistler and J. L. van Hemmen, Neural Computation 12, 385 (2000).

[13] R. P. N. Rao and T. J. Sejnowski, in Advances in Neural
Information Processing Systems, edited by S. A. Solla, T. K. Leen, and K. R. Müller (Cambridge, MA:MIT Press, 2000), vol. 12, pp. 164-170.

[14] P. Munro and G. Hernandez, in Advances in Neural Information Processing Systems, edited by S. A. Solla, T. K. Leen, and K. R. Müller (Cambridge, MA:MIT Press, 2000), vol. 12, pp. 150-156.

[15] M. Yoshioka, Physical Review E 65, 011903 (2002).

[16] N. Matsumoto and M. Okada, Neural Computation 14, 2883 (2002), in Advances in Neural Information Processing Systems, edited by T. G. Dietterich, S. Becker and Z. Ghahramani (Cambridge, MA:MIT Press, 2002), vol. $14,245-252$.

[17] A. Herz, B. Sulzer, R. Kuhn, and J. L. van Hemmen, Biological Cybernetics 60, 457 (1989).

[18] W. Gerstner, R. Ritz, and J. L. van Hemmen, Biological Cybernetic 69, 503 (1993).

[19] L. F. Abbott and K. I. Blum, Cerebral Cortex 6, 406 (1996).

[20] M. Okada, Neural Networks 9, 1429 (1996).

[21] S. Amari and K. Maginu, Neural Networks 1, 63 (1988).

[22] K. Mimura, T. Kimoto, and M. Okada, condmat/0207545.

[23] K. Kitano and T. Aoyagi, Journal of Physics A: Mathematical and General 31, L613 (1998). 